\title{
Automated Leaf Alignment and Partial Shape Feature Extraction for Plant Leaf Classification
}

\author{
Laith Emad Hamid and S.A.R. Al-Haddad \\ Department of Computer and Communication System Engineering, Faculty of Engineering, \\ Universiti Putra Malaysia (UPM), Selangor, Malaysia
}

Received 17th June 2017; accepted 30th March 2019

\begin{abstract}
The last few decades have witnessed various approaches to automate the process of plant classification using the characteristics of the leaf. Several approaches have been proposed, and the majority focused on global shape features. However, one challenge that faces this task is the high interclass similarity amongst the leaves of different species in terms of the global shape. Furthermore, there always has been an obstacle against full automation as several approaches require user intervention to align the leaf. Therefore, a new set of Quartile Features (QF) is proposed in this paper to describe the partial shape of the leaf, in addition to an automated alignment approach to automate the system. The QF are extracted from the horizontal and vertical leaf quartiles to describe the partial shape of the leaf and the relations among its parts. The well-known Flavia dataset has been selected for the evaluation of the proposed system. The experimental results indicate the ability of the proposed alignment algorithm to align leaves with different shapes and maintain a correct classification accuracy regardless of the orientation of the input leaf samples. Furthermore, the proposed QF indicated promising results by increasing the accuracy of the classification by a range of approximately $26 \%$ to $30 \%$ when combined with Hu's Moment Invariants, using k-fold cross-validation technique.
\end{abstract}

Key Words: Plant leaf classification, quartile features, partial shape, feature extraction, leaf alignment.

\section{Introduction}

It is no mystery why plants are important in our lives. They are the primary source of food to almost all humans and animals. They provide Oxygen, without which there would be no life on this planet. Plants also have a great importance in medicine, clothing, fuel, and many other industries and fields of life. Therefore, it is essential to understand plants and classify them.

Plant classification, or taxonomy, is the field of science that studies the morphological features of several parts of the plants and arranges them in hierarchical categories. Parts such as the leaf, the bark, the flower, and the seedlings are used by botanists to differentiate one species from another. Among those parts, the leaf has received the most attention in the field of computer vision due to its approximately 2-Dimensional shape [1] compared to other parts like the flower. Plants are also preferred because collecting them will not cause serious

Correspondence to: laithemad@gmail.com

Recommended for acceptance by Angel Sappa

https://doi.org/10.5565/rev/elcvia.1143

ELCVIA ISSN: $1577-5097$

Published by Computer Vision Center / Universitat Autonoma de Barcelona, Barcelona, Spain 
harm to the plant or the crop as they will wither and fall off eventually. Therefore, many computerized leaf classification approaches are performed by extracting the features of the leaves.

In this paper, we focused on automating the alignment of the leaf and extracting partial shape features towards implementing a fully automated classification system. Therefore, an automated alignment algorithm is proposed to eliminate the need for human intervention, usually required in the pre-processing stage, to extract partial shape features that are based on slicing the leaf into multiple parts.

A new set of Quartile Features (QF) is also proposed in this research to describe the partial shape of the leaf and the relations among different consecutive parts. These features are extracted by slicing the leaf image into quarters that we chose to refer to as quartiles. Once the leaf is automatically aligned with the horizontal axis, a set of 12 QF is extracted from the areas and the perimeters of 4 Horizontal Quartiles (HQ) and 4 Vertical Quartiles (VQ).

\section{Related Work}

A considerable amount of research has been conducted to recognize the plants based on the traits of their leaves. Different features and classifiers have been proposed. However, not much attention has been given to automating the alignment of the leaf in the pre-processing stage. Such automation is required for automating the system, and for ensuring a correct extraction of the partial shape features that are usually based on slicing the leaf. It is also essential for iterative experiments and extensive testing. Therefore, some of the current approaches use the orientation of the leaf to align it automatically [2, 3]. This alignment is accomplished by measuring the orientation of the leaf, represented by the angle between the horizontal axis and the major axis, and rotating the image according to that angle. However, this method is limited to the leaves with an elongated shape as the main vein is usually the same as the major axis of the leaf. Wide or lobed leaves, on the other hand, are the challenging part. Using the orientation to align those leaves usually results in misalignments as their main vein is usually shorter than the major axis. This issue is the main reason that the current approaches [4-6] require the help of the user to align the leaf by selecting certain points on the lamina. On the other hand, manual rotation is also performed in some systems before processing the leaf images to tackle the alignment issue [7].

After the pre-processing stage, feature extraction is the next key stage that determines the success or the failure of the classification process. The extracted features must be robust and discriminative to differentiate among different species. Most of the current systems focus on global shape features to describe the traits of the leaf numerically. Digital Morphological Features (DMF) represent one of the most used approaches to describe the global shape by extracting features from basic geometric measures. DMF have been presented in [4] as an input to a Probabilistic Neural Network (PNN) to classify 1800 leaves from 32 species in the dataset named Flavia. DMF were later adopted and extended in a variety of approaches to classify the leaves. In [8], a Support Vector Machine (SVM) is employed with Principal Component Analysis (PCA) to reduce the dimensionality of the DMF into 5 principal components. In [5], an Artificial Neural Network (ANN) is used to classify 20 species. The system extracts width and angle ratios along with other DMF at different distances across the main vein. ANN classifier is also used in [2], using a combination of DMF with Fourier descriptors and a Shape-Defining Feature (SDF). Another form of DMF is proposed in [9], using a standardized matrix representation of the leaf shape. The features are represented by different measures and angles at different distances from the tip and the base points.

Hu's Moment Invariants (MI) represent another category of global shape descriptors that were introduced by $\mathrm{Hu}[10]$. Those moments have been used by several researchers due to their invariance to translation, rotation, and scaling. Hu's MI are combined with DMF in [11] to classify 20 species using a moving median center hypersphere classifier. In [12], MI are combined with Centroid Radii to classify 3 types of plants. MI are also employed in [13] as an input to an SVM classifier, and in [14] to differentiate the weed from the crop using a Nearest Neighbor Classifier (1-NN). Zernike Moment Invariants (ZMI) are another category of invariant moments. ZMI have been investigated by researchers for plant classification. In [15], ZMI are combined with DMF using a PNN classifier, whereas in [16], ZMI are combined with other shape, color, and texture features for leaf classification. 
The aforementioned features represent the most commonly used global shape descriptors. However, since many species share the same global shape, those features are unable, in certain cases, to overcome the global shape similarity among different species. Therefore, partial shape approaches have also been proposed by some researchers. In [7], Half-Leaf Features (HLF) are extracted from two half-regions of the leaf using a PNN classifier. In [17], a leaf classification technique based on local representation of leaf parts was proposed. The leaves are aligned vertically then divided into 3 parts based on 3 botanical definitions as apical, basal, and middle parts. This approach proposed an automated alignment method based on the base detection algorithm in [18]. However, this algorithm requires the existence of the petiole for a successful insertion point detection. In [6], a set of partial features is extracted by slicing across the horizontal axis after aligning the leaf horizontally. The features are referred to as Leaf Width Factor (LWF) and are calculated as ratios of the length of each slice to the length of the major axis. This approach, as mentioned earlier, relies on the user to select the base point and other reference points for segmenting and aligning the leaf. Another partial shape approach was proposed in [19], using a sub-classifier strategy based on the local and the global shape criteria in the flora books. Four sets of attributes have been considered to extract botanical labels input into a Random Forests classifier (RF) to identify the species of a plant.

In addition to the aforementioned approaches, several other methods have been presented. Contour Centroid Distance (CCD) has been utilized in [20] to calculate the distance from the leaf centroid to a number of sample points on the leaf contour. A similar distance-based approach also appears in [21], using the distance between the centroid and the axis of least inertia. The advantage of the latter method is the invariance to scale, translation, and rotation. The contour of the leaf was also used for classification using different edge extraction operators to extract the shape of the leaf boundary $[22,23]$.

\section{Proposed System}

This system is designed to classify simple leaves captured against a white background. The petioles of the leaves must be removed before processing the images. After the RGB leaf image is acquired, the image is converted into grayscale format, and then binarized using Otsu's thresholding algorithm [24]. After that, a series of basic morphological operations are performed to remove the noise and fill in the holes. After that, the image undergoes the following three main stages:

\subsection{Automated Leaf Alignment}

This stage of image pre-processing is required to align the leaf horizontally according to its main vein before extracting the features. The steps to perform the alignment are described as follows:

a. The initial orientation of the leaf is calculated as the angle between the horizontal axis and the major axis of the leaf. The major axis of an irregular shape can be defined as the major axis of the ellipse that has the same second-moments as that shape [1].

b. The resulting image from step (a) is cropped to its bounding box to fill the entire image frame and eliminate any redundant background pixels outside the bounding box.

c. The image is then divided in half twice, once vertically and another horizontally. A logical AND operator is applied to the upper half of the leaf and the lower half flipped vertically to obtain the upper-lower similarity measure $\left(\mathrm{S}_{1}\right)$. The same is done to the right half and the left half flipped horizontally to calculate the left-right similarity measure $\left(\mathrm{S}_{2}\right)$.

d. The two measures are compared and a decision is made based on the comparison result and the condition that the Aspect Ratio (AR) is higher than a certain threshold (T). The comparison with the aspect ratio is necessary to ensure that only the wide and lobed leaves are rotated and prevent the misalignment of the elongated leaves which can already be aligned using the traditional orientation method. Based on the comparison, a decision is made. If the left-right similarity measure (S2) is larger than the similarity between the upper and the lower halves (S1), the leaf is rotated 90 degrees. Otherwise, it will be kept aligned horizontally.

e. At this stage, the leaf is horizontally aligned with its main vein aligned along the horizontal axis. The image is bisected vertically one more time and the areas of both halves are compared again. If the area 
of the left half $\left(\mathrm{A}_{\text {left }}\right)$ is greater than that of the right half $\left(\mathrm{A}_{\text {right }}\right)$, the leaf is left intact. Otherwise, it is rotated 180 degrees to obtain the final horizontally aligned leaf, with its base on the left side of the image and its apex on the right side.

The flowchart of the proposed automated alignment algorithm is illustrated in Figure 1. A leaf of the species Acer palmatum in Flavia dataset [2] is shown as an example of a wide, lobed leaf alignment.

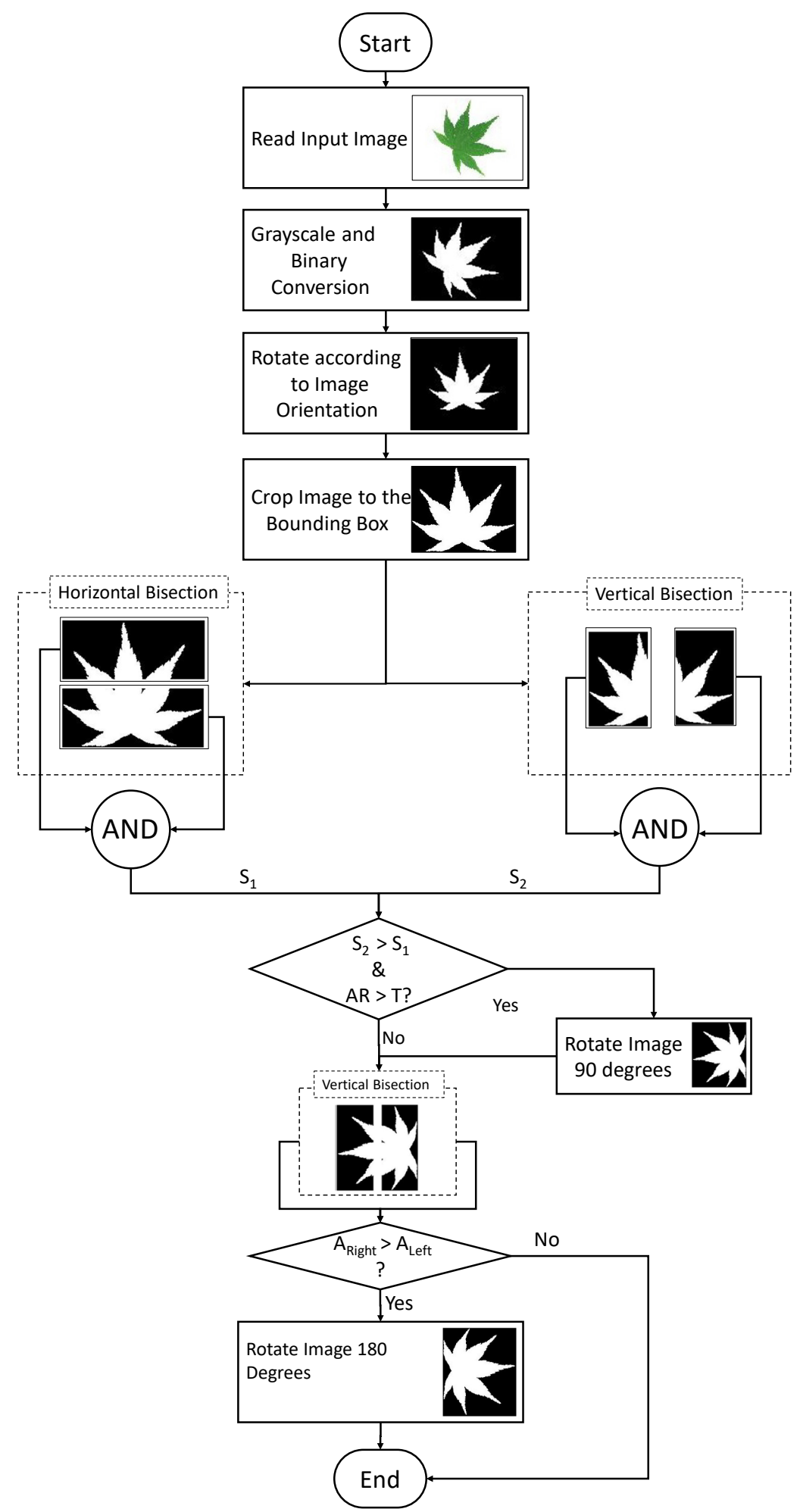

Figure 1: Flowchart of the proposed automated leaf alignment algorithm 


\subsection{Feature Extraction}

Feature extraction is a key part of any classification system since it determines the accuracy of the classification. The extracted features must be robust and discriminative enough to differentiate different species. Thus, the features must reflect a high intraclass similarity within the leaves of the same species, in addition to a low interclass similarity with other species [26]. Therefore, two categories of features are extracted in this research. The first is a proposed set of partial shape features and the second is the well-known Hu's MI that are extracted to evaluate and compare the rotation-invariance and the efficiency of the proposed features.

\section{Quartile Features (QF):}

This paper proposes a new set of features that we refer to as Quartile Features (QF). These features are meant to express the partial shape of the leaf and the relations among its consecutive quartile parts to overcome the global shape similarity issue. After aligning the image horizontally using the proposed alignment algorithm, the binary leaf image is divided into 4 equal Horizontal Quartiles (HQ) and 4 equal Vertical Quartiles (VQ), as shown in Figure 2.

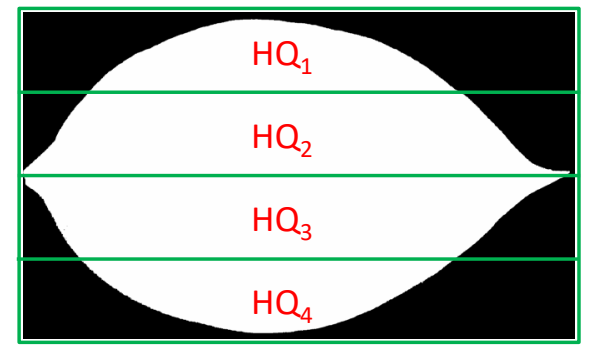

(a)

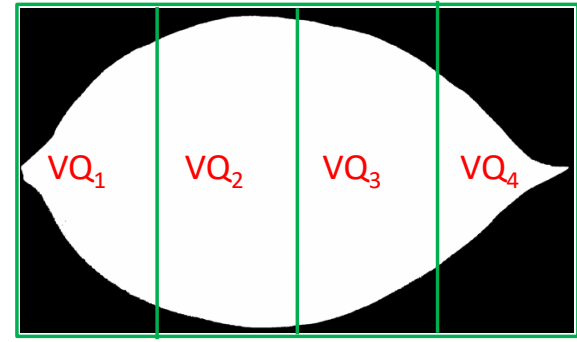

(b)

Figure 2: Quartile parts of the leaf: (a) Horizontal Quartiles (b) Vertical Quartiles

The area and the perimeter of each quartile are calculated, and a set of 6 Horizontal Features (HF) is extracted. The first three Horizontal Features (HF) represent the quartile area features and are defined as follows:

$$
\begin{aligned}
& H F_{1}=A_{H Q 1} / A_{H Q 2} \\
& H F_{2}=A_{H Q 2} / A_{H Q 3} \\
& H F_{3}=A_{H Q 3} / A_{H Q 4}
\end{aligned}
$$

Where $H F_{i}$ denotes the $i^{\text {th }}$ horizontal feature, while $A_{H Q 1}, A_{H Q 2}, A_{H Q 3}$, and $A_{H Q 4}$ represent the area of each $\mathrm{HQ}$ of the leaf, starting from the top quartile, respectively. Similarly, perimetric quartile features are calculated from the perimeters of each two consecutive HQ as follows:

$$
\begin{aligned}
& H F_{4}=P_{H Q 1} / P_{H Q 2} \\
& H F_{5}=P_{H Q 2} / P_{H Q 3} \\
& H F_{6}=P_{H Q 3} / P_{H Q 4}
\end{aligned}
$$

Where $P_{H Q 1}, P_{H Q 2}, P_{H Q 3}$, and $P_{H Q 4}$ represent the perimeter of each horizontal quartile of the leaf, starting from the top quartile, respectively. In a similar fashion, 6 Vertical Features (VF) are calculated from the areas and the perimeters of the VQ. The first three VF represent vertical area features, and are calculated as follows:

$$
\begin{aligned}
& V F_{1}=A_{V Q 1} / A_{V Q 2} \\
& V F_{2}=A_{V Q 2} / A_{V Q 3} \\
& V F_{3}=A_{V Q 3} / A_{V Q 4}
\end{aligned}
$$


Where $V F_{i}$ denotes the $i^{\text {th }}$ vertical feature, and $A_{V Q 1}, A_{V Q 2}, A_{V Q 3}$, and $A_{V Q 4}$ represent the area of each VQ of the leaf, starting from the left, respectively. Finally, three vertical perimetric features are extracted as follows:

$$
\begin{aligned}
& V F_{4}=P_{V Q 1} / P_{V Q 2} \\
& V F_{5}=P_{V Q 2} / P_{V Q 3} \\
& V F_{6}=P_{V Q 3} / P_{V Q 4}
\end{aligned}
$$

Where $P_{V Q 1}, P_{V Q 2}, P_{V Q 3}$, and $P_{V Q 4}$ represent the perimeter of each VQ of the leaf, starting from the left, respectively.

\section{Hu's Invariant Moments:}

In order to evaluate the efficiency of the proposed QF, Hu's MI are extracted in this research. Those seven moments have been proposed by $\mathrm{Hu}$ [3], to describe the global shape of an object. The main advantage of those moments is their invariance to rotation, scaling, and translation. The moment $(m)$ of order $(p, q)$ for an image $I(x, y)$ can be calculated using the following equation:

$$
m_{p q}=\sum_{x} \sum_{y}\left[x^{p} y^{q} I(x, y)\right]
$$

The moments resulting from Equation 13 are called raw moments which are not invariant to transformations. To achieve the invariance several calculations are performed to obtain the final seven moments denoted $\phi_{i}$ where (i) represents the order of the moment and ranges from 1 up to 7.

Due to space limitation, the derivation of the final moments is not repeated in this paper. However, the formulas that we adopted for extracting these moments can be found in [4], to obtain the final seven moments that represent an invariant signature of the global shape of the leaf.

\subsection{Classification}

After all the features are extracted, they are combined into one feature vector and fed into the classifier. In this research, a $k$-Nearest Neighbor classifier $(k$-NN) is employed. This classifier predicts the class of an unknown sample by computing the multidimensional distance between the query feature vector and all the vectors stored in the database. The predicted species is determined based on the majority votes of $k$ neighbors of the query. In this research, the first nearest neighbor is selected for the classification, based on the results of experimenting with different $k$ values, which has also been recommended in the findings of previous researchers that indicated higher accuracy rates compared to a higher number of neighbors [5]-[8]. The distance measure used is City Block which is calculated as follows:

$$
d=\sum_{i}^{n}\left|p_{i}-q_{i}\right|
$$

Where $(d)$ represents the distance between the query $(q)$ and a reference point $(p)$ in the database, while $(n)$ is the total number of features and $(i)$ is the index of the feature in the feature vector. The feature vectors are normalized before being input to the classifier, due to their inconsistent ranges of values. The accuracy rate of the classification is calculated as in the following formula:

$$
\text { Accuracy }(\%)=\frac{\text { Number of Correctly Classified Samples }}{\text { Total Number of Testing Samples }} \times 100 \%
$$

\section{$4 \quad$ Experimental Results}

All the experiments in this research have been conducted using Flavia dataset, which contains 1907 leaf samples from 32 species. This dataset was selected as a benchmark by several researchers, hence, it was selected to evaluate the performance of the proposed system as well. 


\subsection{Automated Alignment Results}

In order to evaluate the efficiency of the proposed automated alignment algorithm, 10 different samples from each of the 32 species in the dataset were used for testing. Each leaf image was rotated 4 times by multiples of 45 degrees, i.e., $45^{\circ}, 90^{\circ}, 135^{\circ}$ and $180^{\circ}$. The alignment algorithm was applied to all the samples for all four angles, and the comparison was done visually by the authors between each rotated image and its aligned version as illustrated in Figure 3.
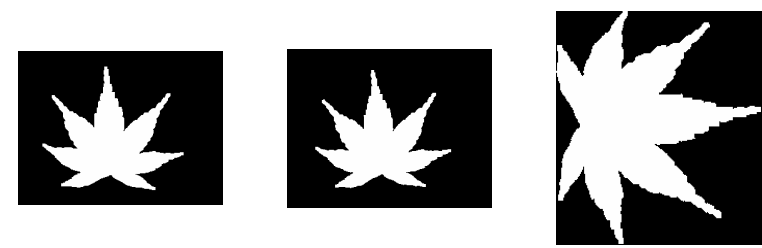

(a) Input Image rotated by 0 degrees
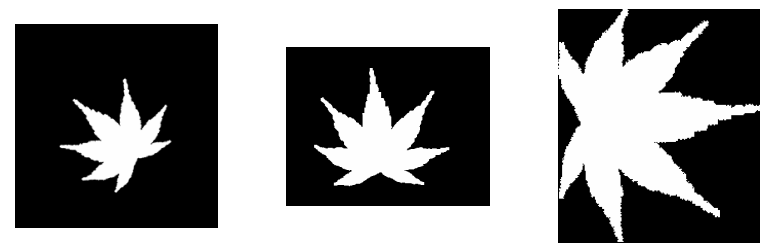

(b) Input Image rotated by 45 degrees
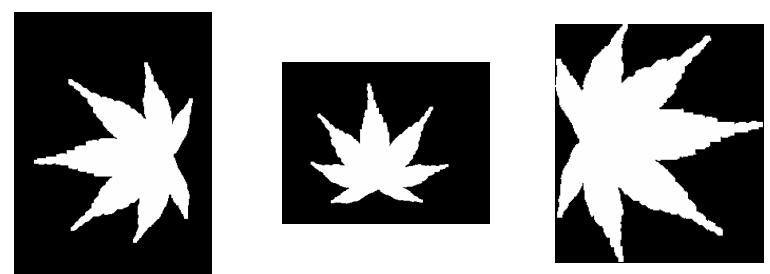

(c) Input Image rotated by 90 degrees
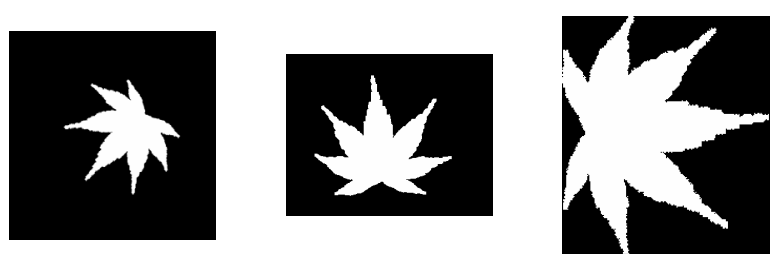

(d) Input Image rotated by 135 degrees
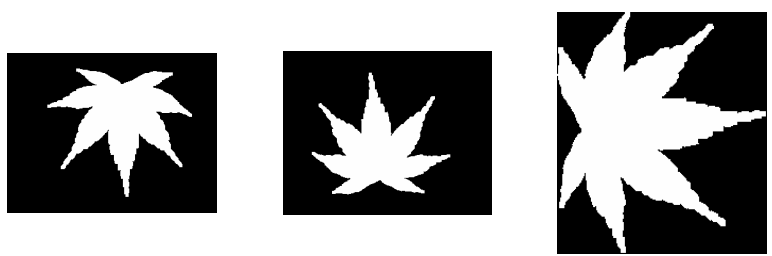

(e) Input Image rotated by 180 degrees

Figure 3: Alignment results for a lobed leaf: (Left) rotated input image, (Middle) alignment result using the conventional orientation approach, (Right) alignment result using the proposed algorithm

Based on the visual assessment, the proposed algorithm was able to align the majority of the leaf samples correctly. The output aligned images indicated that the proposed algorithm is able to align several leaves regardless of the orientation of the input image (See Figure 4). Furthermore, the proposed algorithm was capable of aligning the challenging lobed leaves $100 \%$ of the times correctly, which is an improvement over the conventional alignment approach. However, some samples from 5 species were misaligned when rotated by 
certain angles due to the irregularity of their shapes that caused a noticeable asymmetry across the main vein, thus, affecting the similarity measure that the proposed algorithm is based on.
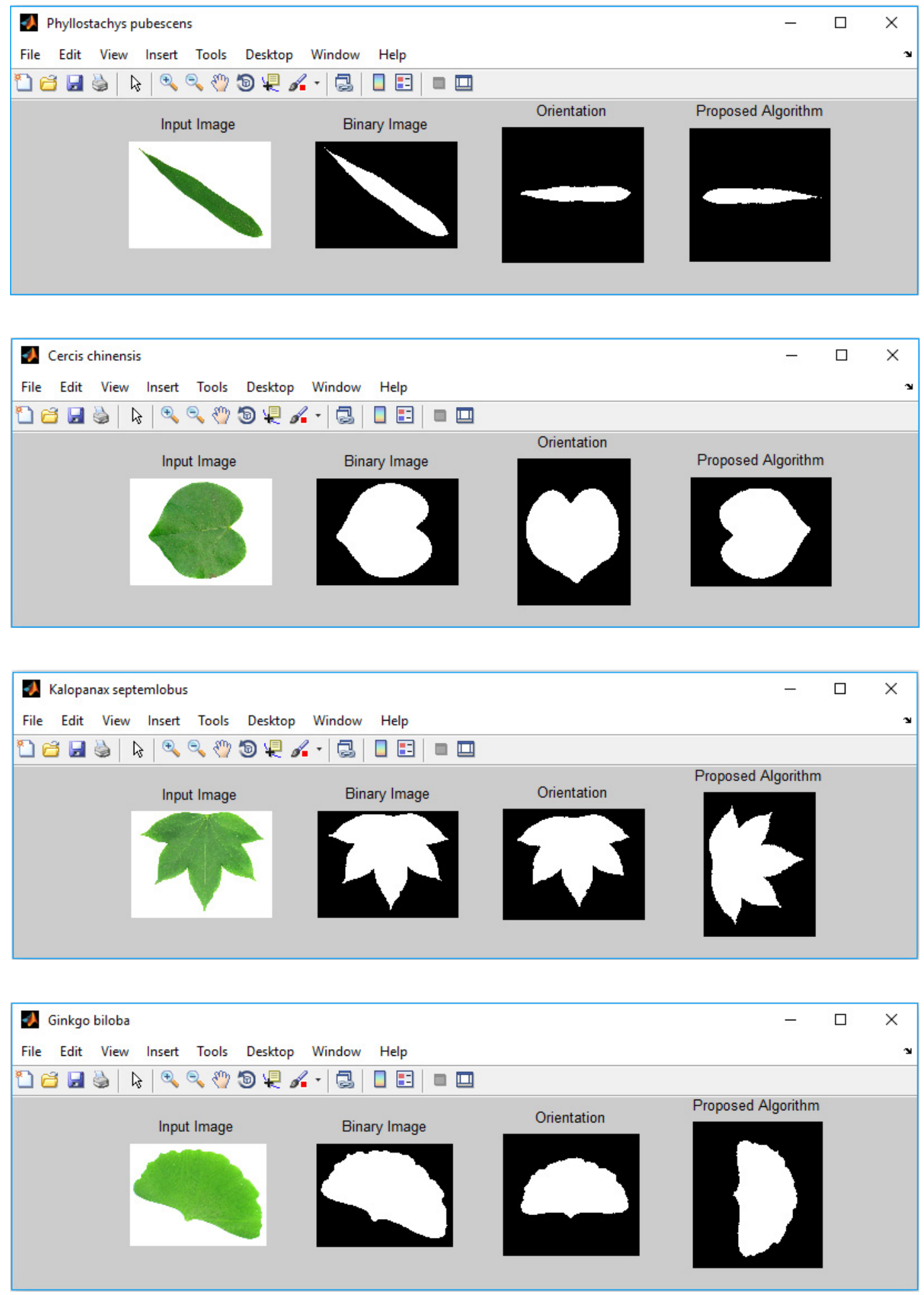

Figure 4: Alignment results for different leaf shapes

To assess the efficiency of the proposed alignment algorithm further, an experiment was conducted to classify the leaves using the proposed QF. In this experiment, $25 \%$ of the data were used as a training set, while the remaining $75 \%$ were used for testing. The testing images were rotated by multiples of 45 degrees to investigate the impact of the proposed alignment on the accuracy using QF. The following three cases were studied in this experiment: 
- Each sample in the testing set was rotated and input to the classifier without being aligned.

- The testing samples were rotated and then aligned before being input to the classifier, using the orientation of the major axis approach, mentioned earlier.

- The testing samples were rotated and then aligned using the proposed alignment algorithm.

The results of this experiment are shown in Figure 5.

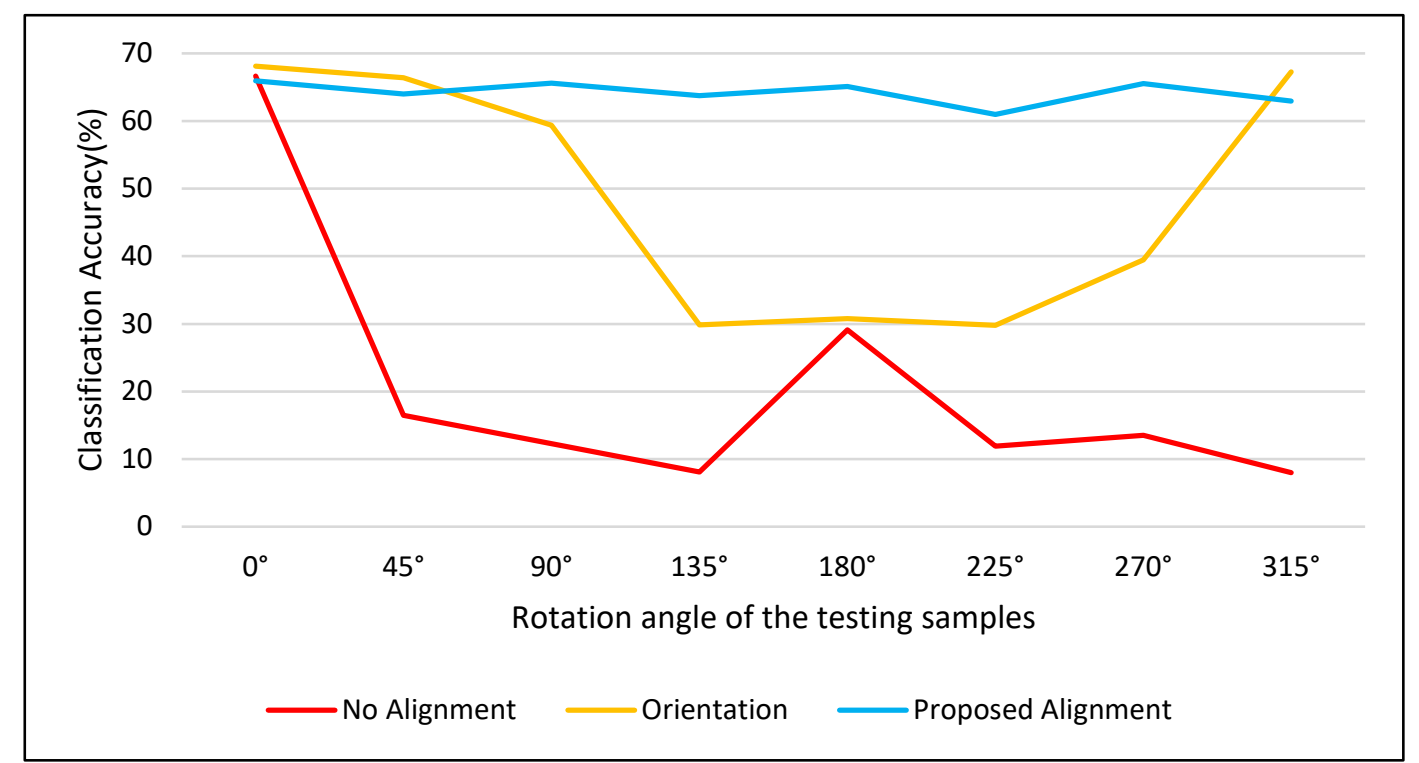

Figure 5: Accuracy rates for different orientations of the testing samples

Figure 5 shows an expected drop in the accuracy when no alignment is applied as the extracted QF depend solely on the orientation of the leaf. On the other hand, it can be seen that the proposed alignment method outperformed the conventional orientation approach when the testing samples were rotated by 90 degrees or higher. This decrease in the accuracy is attributed to the wide and lobed samples that are misaligned when rotated, and thus, leading to an incorrect feature extraction that affects the classification accuracy negatively.

Therefore, despite the aforementioned misalignments that faced the proposed algorithm in aligning a few samples, the algorithm is still capable of aligning the majority of the wide and lobed leaves which can be considered an advantage over the conventional orientation approach. Furthermore, the proposed method provides a good level of rotation invariance for extracting partial shape features by maintaining the accuracy with a very slight variation as can be seen in the previous figure.

\subsection{Feature Extraction Results}

To study the robustness of each individual extracted feature, the same aforementioned dataset partitioning is used. The following experiments were conducted using $25 \%$ of the total images for training, i.e. 480 samples, while the remaining 1427 samples were used for testing the features. The reason for using less training samples is to evaluate the ability of the features to differentiate between different species without relying on large numbers of training samples, which may lead to high accuracy rates regardless of the features' robustness.

\section{Quartile Features:}

Each individual QF has been used to classify the same set of testing samples, and the classification accuracy rates were recorded accordingly. Different combinations of HF and VF have also been investigated to determine the impact of the different features on the classification. The accuracy rates of using each individual HF and VF are shown in Tables 1 and 2 respectively. 


\begin{tabular}{lc}
\hline Feature & Accuracy (\%) \\
\hline $\mathrm{HF}_{1}$ & 23.55 \\
$\mathrm{HF}_{2}$ & 20.25 \\
$\mathrm{HF}_{3}$ & 23.34 \\
$\mathrm{HF}_{4}$ & 23.13 \\
$\mathrm{HF}_{5}$ & 20.88 \\
$\mathrm{HF}_{6}$ & 23.27 \\
\hline
\end{tabular}

Table 1: Accuracy rates using Horizontal Features individually

\begin{tabular}{lc}
\hline Feature & Accuracy $(\%)$ \\
\hline $\mathrm{VF}_{1}$ & 24.39 \\
$\mathrm{VF}_{2}$ & 23.62 \\
$\mathrm{VF}_{3}$ & 28.10 \\
$\mathrm{VF}_{4}$ & 23.69 \\
$\mathrm{VF}_{5}$ & 21.51 \\
$\mathrm{VF}_{6}$ & 27.47 \\
\hline
\end{tabular}

Table 2: Accuracy rates using Vertical Features individually

The results shown in the previous two tables indicate low accuracy rates with a slightly varying range for each individual HF and VF. However, by combining different features, a significant increase in the accuracy was obtained. The results in Table 3 show the accuracy rates using different numbers and combinations of features. The results also indicate that the VF combined are more discriminative than the HF combined, with an accuracy rate of $64.33 \%$. This is an expected result due to the natural symmetry across the main vein of the leaf that makes adjacent vertical quartiles more variant, i.e. more discriminative, than horizontal quartiles.

\begin{tabular}{lccc}
\hline Features Description & $\begin{array}{c}\text { No. of } \\
\text { Features }\end{array}$ & Feature Combination & Accuracy (\%) \\
\hline Horizontal Area Features & 3 & $\mathrm{HF}_{1}-\mathrm{HF}_{3}$ & 32.24 \\
Horizontal Perimetric Features & 3 & $\mathrm{HF}_{4}-\mathrm{HF}_{6}$ & 32.37 \\
Horizontal Features & 6 & $\mathrm{HF}_{1}-\mathrm{HF}_{6}$ & 40.85 \\
Vertical Area Features & 3 & $\mathrm{VF}_{1}-\mathrm{VF}_{3}$ & 48.91 \\
Vertical Perimetric Features & 3 & $\mathrm{VF}_{4}-\mathrm{VF}_{6}$ & 45.13 \\
Vertical Features & 6 & $\mathrm{VF}_{1}-\mathrm{VF}_{6}$ & 64.33 \\
Area Features & 6 & $\left(\mathrm{HF}_{1}-\mathrm{HF}_{3}\right)+\left(\mathrm{VF}_{1}-\mathrm{VF}_{3}\right)$ & 59.71 \\
Perimetric Features & 6 & $\left(\mathrm{HF}_{4}-\mathrm{HF}_{6}\right)+\left(\mathrm{VF}_{4}-\mathrm{VF}_{6}\right)$ & 52.35 \\
Quartile Features & $\mathbf{1 2}$ & $\left(\mathbf{H F}_{\mathbf{1}}-\mathbf{H F}_{\mathbf{6}}\right)+\left(\mathbf{V F}_{\mathbf{1}}-\mathbf{V F}_{\mathbf{6}}\right)$ & $\mathbf{6 6 . 2 9}$ \\
\hline
\end{tabular}

Table 3: Accuracy rates using different combinations of $\mathrm{HF}$ and $\mathrm{VF}$

Moreover, by analyzing the results in Table 3, it can also be deduced that the features extracted from the areas of the quartiles have outperformed the perimetric features for both vertical and horizontal categories. However, combining all 12 features from all categories yielded the highest rate in this experiment. Therefore, all twelve features were selected as the final feature vector for the overall performance evaluation experiment, discussed in Section 4.3.

\section{Hu's Invariant Moments:}

These features have been extracted to provide an understanding and a comparison of the efficiency of proposed QF in terms of rotation invariance, in addition to investigating the feasibility of using QF for leaf classification along with other features. The results of each individual MI in classifying the same aforementioned testing set are shown in Table 4. It can be seen from the results that the proposed QF approach 
combined with the proposed alignment algorithm has outperformed Hu's MI in terms of the classification accuracy. It can also be noticed that the moments up to the third order contribute to most of the classification accuracy as the remaining four moments did not add a significant increase.

\begin{tabular}{lc}
\hline Hu's Invariant Moment Order & Accuracy (\%) \\
\hline $1^{\text {st }}$ & 36.16 \\
$2^{\text {nd }}$ & 37.21 \\
$3^{\text {rd }}$ & 26.56 \\
$4^{\text {th }}$ & 27.33 \\
$5^{\text {th }}$ & 30.20 \\
$6^{\text {th }}$ & 26.35 \\
$7^{\text {th }}$ & 26.91 \\
$1^{\text {st }} 2^{\text {nd }}$ & 48.56 \\
$1^{\text {st }}-3^{\text {rd }}$ & 50.11 \\
All 7 moments & $\mathbf{5 0 . 8 1}$ \\
\hline
\end{tabular}

Table 4: Accuracy rates using Hu's Invariant Moments

\subsection{Performance Evaluation}

An extensive k-fold cross-validation experiment was conducted to evaluate the overall performance of the proposed system. This technique can provide a realistic and an unbiased evaluation as the entire dataset is partitioned randomly into $k$ equal-sized subsets of samples. The number of folds $(k)$ selected in this experiment ranges from 2 up to 20 folds. For each fold, the algorithm was run 100 times and the results were recorded.

First, the experiment was conducted using QF as the sole feature vector to the 1-NN classifier. The overall minimum, maximum, and average accuracy rates of all the 100 runs for each fold are illustrated in Figure 6. By considering the average accuracy rates, the results seem promising. The proposed QF were able to correctly classify a range of $70.5 \%$ to $77.92 \%$ of the testing samples using the proposed 12 features alone. This indicates that the QF are discriminative in terms of the partial shape of the leaf and can be utilized for leaf classification. Furthermore, by conducting the same experiment using Hu's MI as the input feature vector to the classifier, it can be noticed that the proposed partial shape approach has outperformed the global shape descriptors represented by Hu's MI. The accuracy rates were considerably lower using the latter approach as the average accuracy ranged from $53.9 \%$ to $62.39 \%$ for the $2^{\text {nd }}$ and $20^{\text {th }}$ folds, respectively (Refer to Figure 7 ).

Furthermore, by conducting the same experiment a third and final time, using a combination of $12 \mathrm{QF}$ and $7 \mathrm{MI}$. The results of the hybrid approach indicated a significant increase in accuracy, achieving average accuracy rates ranging from $84.32 \%$ to $89.13 \%$. Thus, this is an improvement by a range of $26.38 \%$ to $30.42 \%$ compared to using Hu's global shape descriptors alone. Furthermore, several higher rates were also recorded as more training samples were used with each increment in the number of folds as can be seen in Figure 8.

Therefore, it can be deduced that the proposed Quartile Features and the automated alignment approach can provide a promising level of classification accuracy, especially when combined with other global shape descriptors. Moreover, the proposed system provides an acceptable level of invariance to rotation that allows the extraction of other partial features in future research work. 


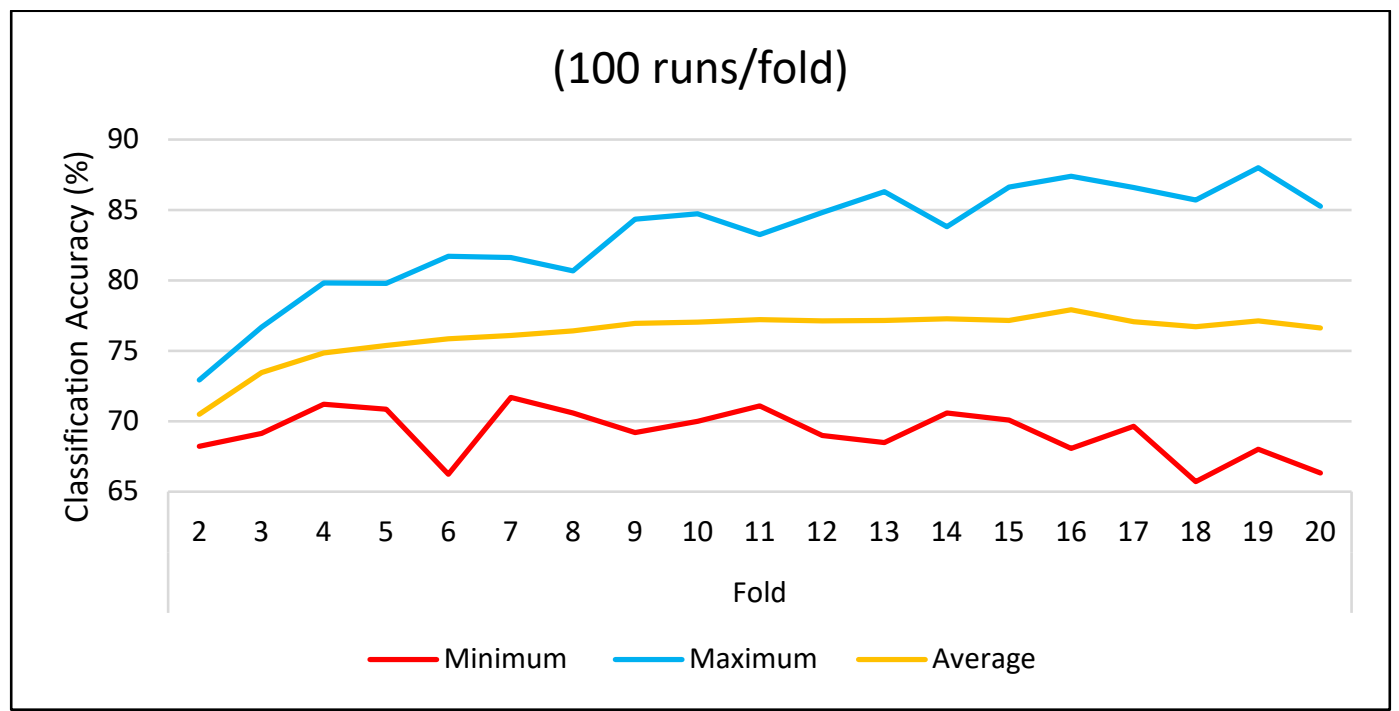

Figure 6: K-fold cross-validation using $12 \mathrm{QF}$

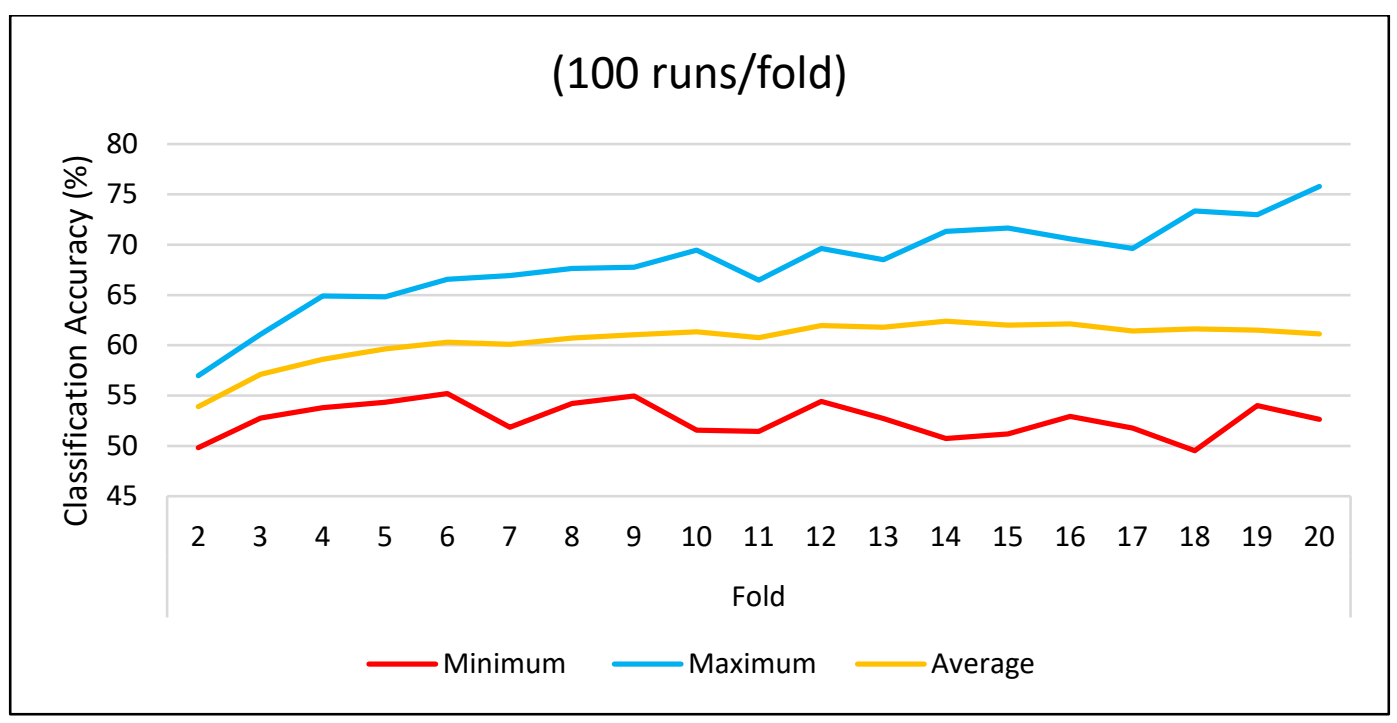

Figure 7: K-fold cross-validation using 7 Invariant Moments

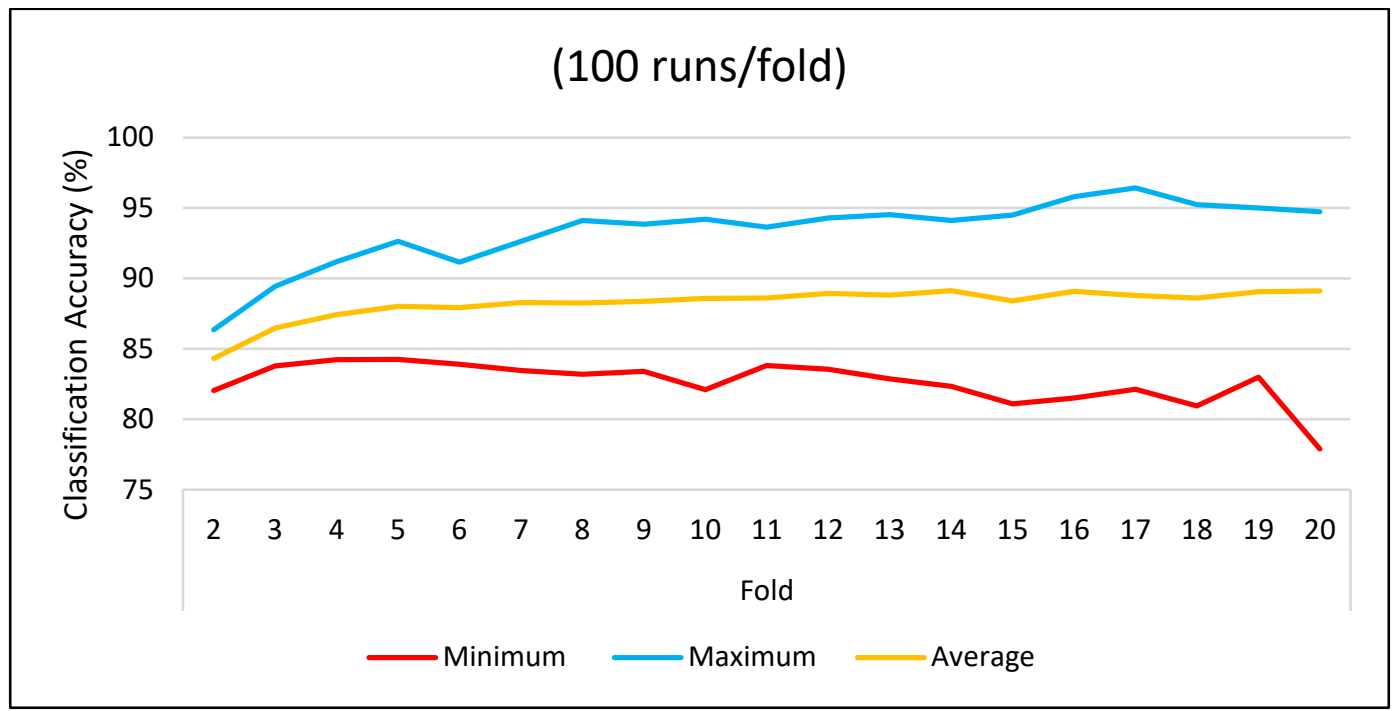

Figure 8: K-fold cross-validation using a hybrid of QF and MI 


\subsection{Performance Comparison}

To establish a realistic and accurate comparison of the proposed approach with the techniques reported in the literature, the experiments conducted using Flavia dataset have been compared. Each method has a different experimental setup in terms of the numbers of training and testing samples. Therefore, the results from our experiments have been compared based on the number of folds $(k)$ that matches the equivalent numbers of training and testing samples for the corresponding methods in the literature as shown in Table 5. The results compared from our experiments are the maximum, minimum, and average classification accuracy rates of 100 runs/fold as graphed in Figure 8.

\begin{tabular}{|c|c|c|c|c|c|c|}
\hline \multirow[b]{2}{*}{ Method } & \multirow[b]{2}{*}{$\begin{array}{c}\text { Leaf } \\
\text { Alignment }\end{array}$} & \multirow[b]{2}{*}{$\begin{array}{l}\text { Accuracy } \\
(\%)\end{array}$} & \multicolumn{4}{|c|}{ Proposed Method } \\
\hline & & & $\begin{array}{c}\text { Equivalent } \\
\text { Number of } \\
\text { Folds }\end{array}$ & $\begin{array}{c}\text { Minimum } \\
\text { Accuracy } \\
(\%)\end{array}$ & $\begin{array}{c}\text { Average } \\
\text { Accuracy } \\
(\%)\end{array}$ & $\begin{array}{c}\text { Maximum } \\
\text { Accuracy } \\
(\%)\end{array}$ \\
\hline DMF in [2] & User & 90.30 & 6 & 83.91 & 88.92 & 91.17 \\
\hline LWF and DMF in [9] & User & 91.41 & 10 & 82.10 & 88.57 & 94.21 \\
\hline HLF in [10] & Manual & 92.50 & 8 & 83.19 & 88.24 & 94.12 \\
\hline $\begin{array}{l}\text { DMF and vein features } \\
\text { using k-NN in [11] }\end{array}$ & $*$ & 78.00 & 6 & 83.91 & 88.92 & 91.17 \\
\hline $\begin{array}{l}\text { DMF and vein features } \\
\text { using SVM in [11] }\end{array}$ & $*$ & 94.50 & 6 & 83.91 & 88.92 & 91.17 \\
\hline DMF and k-NN in [12] & $*$ & 65.10 & 2 & 83.77 & 86.50 & 89.45 \\
\hline ZMI and k-NN in [12] & * & 68.30 & 2 & 83.77 & 86.50 & 89.45 \\
\hline $\begin{array}{l}\text { ZMI, DMF, GLCM, and } \\
\text { color in [13] }\end{array}$ & No & 94.69 & 5 & 84.25 & 88.02 & 92.65 \\
\hline CCD in [14] & Invariant & 82.05 & 4 & 84.24 & 87.43 & 91.17 \\
\hline
\end{tabular}

* indicates that no information was provided regarding the alignment of the leaves

Table 5: Performance comparison with different methods in the literature

The comparison indicates good classification accuracy rates compared to the methods reported in the literature. The proposed technique has also reached maximum classification accuracy rates that outperformed most of the methods included in the comparison table. It can also be seen that the classification accuracy has outperformed DMF and ZMI approaches in [12] and CCD method in [14] including the minimum accuracy rates recorded over 100 runs for the equivalent fold numbers.

On the other hand, two of the methods compared have achieved slightly higher accuracy rates than those of the proposed system. This outcome is expected as the proposed method is using simple partial shape features and MI compared to a combination of color, texture, and shape features in [13]. Similarly, by comparing the proposed method with the DMF approach in [11], the impact of using the more efficient SVM classifier is significant compared to using $\mathrm{k}-\mathrm{NN}$ in the same reference.

Nonetheless, the results are promising, considering that the proposed approach can compete with wellestablished techniques that use various feature categories despite the simplicity of the proposed features and the use of a simple k-NN classifier. Furthermore, the proposed system provides an automated alignment approach for the leaves, which eliminates the need for human intervention and provides the ability to conduct extensive tests and experiments as presented in this paper. Therefore, the proposed method has the potential to achieve higher accuracy rates by using more advanced classifiers and adding more features.

\section{Conclusions and Future Work}

This paper presented a new approach to align the leaf images automatically and extract a new set of partial shape features for plant leaf classification. The proposed alignment algorithm indicated encouraging results by maintaining a range of accuracy with slight variations towards changes in the orientation of the leaves, regardless of the few misalignments that occurred when aligning some irregular or deformed leaves. 
Furthermore, the proposed QF indicated promising results to classify different leaves when used as the sole feature vector. The accuracy was increased even further by including Hu's MI by a range of $26.38 \%$ to $30.42 \%$ compared to using the latter features alone. It is also worth mentioning that the system was implemented using a simple 1-NN classifier. Thus, the horizons are open for more improvements using more robust classifiers or by extracting more partial shape features.

\section{References}

[1] I. Yahiaoui, O. Mzoughi, and N. Boujemaa, "Leaf Shape Descriptor for Tree Species Identification," in 2012 IEEE International Conference on Multimedia and Expo(ICME), 2012, pp. 254-259, doi: 10.1109/ICME.2012.130.

[2] A. Aakif and M. F. Khan, "Automatic classification of plants based on their leaves," Biosyst. Eng., vol. 139, pp. 66-75, 2015, doi: 10.1016/j.biosystemseng.2015.08.003.

[3] J. Chaki, R. Parekh, and S. Bhattacharya, "Plant leaf recognition using texture and shape features with neural classifier," Pattern Recognit. Lett., vol. 58, pp. 61-68, 2015, doi: 10.1016/j.patrec.2015.02.010.

[4] S. G. Wu, F. S. Bao, E. Y. Xu, Y. Wang, Y. Chang, and Q. Xiang, "A Leaf Recognition Algorithm for Plant Classification Using Probabilistic Neural Network," in IEEE International Symposium on Signal Processing and Information Technology, 2007, pp. 11-16, doi: 10.1109/ISSPIT.2007.4458016.

[5] S. Hati and G. Sajeevan, "Plant Recognition from Leaf Image through Artificial Neural Network," Int. J. Comput. Appl., vol. 62, no. 17, pp. 15-18, 2013, doi: 10.5120/10172-4897.

[6] J. Hossain and M. A. Amin, "Leaf Shape Identification Based Plant Biometrics," in IEEE 13th International Conference on Computer and Information Technology (ICCIT), 2010, pp. 458-463, doi: 10.1109/ICCITECHN.2010.5723901.

[7] C. Uluturk and A. Ugur, "Recognition of Leaves Based on Morphological Features Derived From Two Half-Regions," in International Symposium on Innovations in Intelligent Systems and Applications (INISTA), 2012, pp. 1-4, doi: 10.1109/INISTA.2012.6247030.

[8] C. Arun Priya, T. Balasaravanan, and A. S. Thanamani, "An Efficient Leaf Recognition Algorithm for Plant Classification Using Support Vector Machine," in Proceedings - International Conference on Pattern Recognition, Informatics and Medical Engineering (PRIME), 2012, pp. 428-432, doi: 10.1109/ICPRIME.2012.6208384.

[9] L. Gao, X. Lin, M. Zhong, and J. Zeng, "A Neural Network Classifier Based on Prior Evolution and Iterative Approximation Used for Leaf Recognition," in Proceedings of 6th International Conference on Natural Computation (ICNC), 2010, vol. 2, pp. 1038-1043, doi: 10.1109/ICNC.2010.5582971.

[10] M.-K. Hu, "Visual Pattern Recognition by Moment Invariants," IRE Trans. Inf. Theory, vol. 8, pp. 179187, 1962, doi: 10.1109/TIT.1962.1057692.

[11] J.-X. Du, X.-F. Wang, and G.-J. Zhang, "Leaf shape based plant species recognition," Appl. Math. Comput., vol. 185, no. 2, pp. 883-893, 2007, doi: 10.1016/j.amc.2006.07.072.

[12] J. Chaki and R. Parekh, "Plant Leaf Recognition using Shape-based Features and Neural Network classifiers," Int. J. Adv. Comput. Sci. Appl., vol. 2, no. 10, pp. 41-47, 2011, doi: 10.14569/IJACSA.2011.021007.

[13] J. Nesaratnam and C. BalaMurugan, "Identifying Leaf in a Natural Image using Morphological Characters," in IEEE Sponsored 2nd International Conference on Innovations in Information, Embedded and Communication systems (ICIIECS), 2015, pp.1-5, doi: 10.1109/ICIIECS.2015.7193115.

[14] Z. Bo, W. H. Hua, L. S. Jun, M. W. Hua, and Z. X. Chao, "Research on Weed Recognition Method Based on Invariant Moments," in Proceeding of the 11th World Congress on Intelligent Control and Automation, 2014, vol. 11, no. 2012, pp. 2167-2169, doi: 10.1109/WCICA.2014.7053057.

[15] B. S. Harish, A. Hedge, O. Venkatesh, D. G. Spoorthy, and D. Sushma, "Classification of plant leaves using morphological features and Zernike moments," in IEEE International Conference on Advances in Computing, Communications and Informatics (ICACCI), 2013, pp. 1827-1831, doi: 10.1109/ICACCI.2013.6637459.

[16] A. Kadir, L. Nugroho, A. Susanto, and P. I. Santosa, "Experiments of Zernike Moments for Leaf Identification," J. Theor. Appl. Inf. Technol., vol. 41, no. 1, pp. 82-93, 2012.

[17] O. Mzoughi, I. Yahiaoui, N. Boujemaa, and E. Zagrouba, “Advanced Tree Species Identification Using 
Multiple Leaf Parts Image Queries," in 20th IEEE International Conference on Image Processing (ICIP), 2013, pp. 3967-3971, doi: 10.1109/ICIP.2013.6738817.

[18] O. Mzoughi, I. Yahiaoui, and N. Boujemaa, "Extraction of Leaf Parts by Image Analysis," in International Conference Image Analysis and Recognition, 2012, pp. 348-358, doi: 10.1109/ICIP.2013.6738817.

[19] H. Liu, D. Coquin, L. Valet, and G. Cerutti, "Leaf Species Classification Based on a Botanical Shape Sub-Classifier Strategy," in IEEE 22nd International Conference on Pattern Recognition (ICPR), 2014, pp. 1496-1501, doi: 10.1109/ICPR.2014.266.

[20] H. Hajjdiab and I. Al Maskari, "Plant Species Recognition Using Leaf Contours," IEEE Int. Conf. Imaging Syst. Tech., pp. 306-309, 2011, doi: 10.1109/IST.2011.5962205.

[21] K. Mahdikhanlou and H. Ebrahimnezhad, "Plant LeafClassification Using Centroid Distance and Axis of Least Inertia Method," in 22nd Iranian Conference on Electrical Engineering (ICEE), 2014, pp. 1690-1694, doi: 10.1109/IranianCEE.2014.6999810.

[22] M. M. Amlekar, A. T. Gaikwad, and R. R. Manza, "Leaf Shape Extraction For Plant Classification," in International Conference on Pervasive Computing (ICPC) Leaf, 2015, vol. 00, no. c, pp. 0-3, doi: 10.1109/PERVASIVE.2015.7087088.

[23] P. Bhandarkar, H. Doshi, M. Kadakia, and R. Ahmed, "Leaf Identification using Morphology and Structural Decomposition," in International Conference on Signal Processing and Integrated Networks (SPIN), 2014, pp. 125-129, doi: 10.1109/SPIN.2014.6776934.

[24] N. Otsu, "A Threshold Selection Method from Gray-Level Histograms," IEEE Trans. Syst. Man. Cybern., vol. 9, no. 1, pp. 62-66, 1979, doi: 10.1109/TSMC.1979.4310076.

[25] Matlab R2013b Image Processing Toolbox documentation. Mathworks Inc. [Online]. Available: http://www.mathworks.com/help/images/index.html.

[26] S. Prasad, P. Kumar, and R. C. Tripathi, "Plant Leaf Species Identification using Curvelet Transform," in IEEE 2nd International Conference on Computer and Communication Technology (ICCCT), 2011, pp. 646-652, doi: 10.1109/ICCCT.2011.6075212.

[27] X. Gu, J.-X. Du, and X.-F. Wang, "Leaf Recognition Based on the Combination of Wavelet Transform and Gaussian Interpolation," in International Conference on Intelligent Computing, 2005, pp. 253-262, doi: 10.1007/11538059_27.

[28] L. Xun, H. Keyi, W. Liliang, and L. Ping, "Index and recognition for the shape contour of plant leaves," Proc. 2009 WRI Glob. Congr. Intell. Syst. GCIS 2009, vol. 4, pp. 436-440, 2009, doi: 10.1109/GCIS.2009.126.

[29] N. Kumar, P. N. Belhumeur, A. Biswas, D. W. Jacobs, I. Lopez, and J. V.B.Soares, "Leafsnap: A Computer Vision System for Automatic Plant Species Identification," Comput. Vis. (ECCV), pp. 502516, 2012, doi: 10.1007/978-3-642-33709-3_36

[30] A. Bhardwaj, M. Kaur, and A. Kumar, "Recognition of plants by Leaf Image using Moment Invariant and Texture Analysis," Int. J. Innov. Appl. Stud., vol. 3, no. 1, pp. 237-248, 2013. 